\title{
Why the public health sector couldn't create Pokémon Go
}

\author{
Becky Freemana,b,c, Josephine Chau ${ }^{a, b}$ and Seema Mihrshahia,b \\ a Prevention Research Collaboration, School of Public Health, Sydney Medical School, University of Sydney, NSW, Australia \\ ${ }^{b}$ Charles Perkins Centre, University of Sydney, NSW, Australia \\ c Corresponding author: becky.freeman@sydney.edu.au
}

\section{Article history}

Publication date: July 2017

Citation: Freeman B, Chau J, Mihrshahi S.

Why the public health sector couldn't

create Pokémon Go. Public Health

Res Pract. 2017;27(3):e2731724.

https://doi.org/10.17061/phrp2731724

\section{Key points}

- The public health sector should look to popular games, such as Pokémon Go, if it hopes to be successful in using technology to improve health

- Building games that are first focused on pleasure and fun, and then on improving health may be more successful

- Corporations that are harmful to health are quick to exploit popular trends in new media

\begin{abstract}
Pokémon Go has been subject to much attention - from both the players that download the augmented reality game and the news media. Amid the exaggerated media reports, Pokémon Go may have unintended health benefits. Players have reported walking more, spending more time with family, experiencing improvements in their mental health and feeling more connected to their communities. It is hard to imagine public health researchers developing a similar game that is fun, taps into pop culture, reaches a wide target audience, makes use of physical and virtual environments, creates a sense of both competition and community, and has spin-off health benefits. Companies that endanger public health immediately recognised the value of the Pokémon Go app, and exploited it to advertise and promote consumption of unhealthy foods. Public health stakeholders need to develop mobile-based interventions within a framework that embraces pleasure, rewards, participation and community. Public health agencies need to be just as nimble and responsive as companies that are harmful to health, or forever be creating games that nobody plays.
\end{abstract}

\section{Introduction}

Pokémon Go, the "inescapable force of nature"1 that began sweeping the world a year ago, made it near impossible to venture outside without seeing crowds of users happily catching monsters with their smartphones. The augmented reality game was released on 6 July 2016; during its first 20 days on the market, it was estimated to have been downloaded 75 million times ${ }^{2}$ and rapidly gained more daily users than the 10 -year-old social media platform Twitter. ${ }^{3}$ This popculture phenomenon has generated tremendous interest from all sectors of the media, and has been simultaneously portrayed as both the worst and the best thing for the future of humanity - a heavy burden for a relatively simple smartphone application.

\section{What is Pokémon Go?}

Pokémon Go builds on the video game franchise created by Japanese game developer Satoshi Tajiri. In short, it is an augmented reality game, freely available 
for download as a smartphone app on both Android and iOS, which uses time-sensitive global positioning system tracking to detect users' locations. The object of the game is to 'catch' Pokémon (pocket monsters) that appear as virtual creatures in real places. The more users move around to different locations, the more likely they are to come across Pokémon, and there are a number of different types of Pokémon to be caught. The game encourages users to walk to 'PokéStops' (usually landmarks) where they can acquire 'PokéBalls' to catch more Pokémon. Users progress through different levels and, at higher levels, battle each other at virtual gyms.

\section{Unintended health benefits}

Amid the exaggerated news media and social media reports, Pokémon Go appears to have unintended health benefits. Hunting for Pokémon may be contributing to public health by stealth. Anecdotally, trying to "catch 'em all" and hatching eggs appears to have led to players walking more and increasing their daily step counts ${ }^{4}$, families spending hours together outdoors ${ }^{5}$, and fostering of greater social interaction and sense of community. ${ }^{6}$ Users have also reported improvements in mental health conditions, such as depression and anxiety, as a result of playing the game. ${ }^{7}$

The public health sector has so far had limited success in using mobile-based apps and games to successfully change health behaviours. ${ }^{8}$ Equally, the commercial fitness sector, although able to design hugely popular apps and wearable tracking devices, is yet to show that users make permanent changes to their physical activity levels. ${ }^{9}$ Pokémon Go shows that there may be another path to positively affecting health, through the creation of games that appear to have no health focus whatsoever. A recent study shows that there are short-term increases in step counts among Pokémon Go players across sex, age and body mass index categories. ${ }^{10}$

It is hard to imagine public health researchers setting out to develop a game that is fun, taps into pop culture, reaches a wide target audience, makes use of physical and virtual environments, and creates a sense of both competition and community, and then waiting to see whether there are any spin-off health benefits. This is the opposite of the approach currently employed, where the 'behaviour' of focus is often the only driver considered in development of health-related games. The difference may help to explain why so many of these mobile-based health behaviour change interventions fail. ${ }^{11}$ If, instead, games were developed based on a model other than health behaviour change, there may be a much higher chance of achieving health gains. Public health should turn its focus to developing mobile-based interventions within a framework that embraces pleasure, rewards, participation and community.

\section{Opportunity for partnership}

Although public health researchers may not have a high level of knowledge about designing games for health promotion, they do have expertise in evaluating and studying the impacts of games and associated media interest through a health-focused lens. Similar to the situation with wearable technologies, where scientists have been granted access to users' raw data, scientists should take a proactive approach, and engage with game makers and other stakeholders to form partnerships for better health understanding. For example, physical activity researchers could examine how many steps Pokémon Go players accumulate each day over several weeks or months and track long-term impacts of the game on physical activity. Health communications researchers could study Instagram posts about Pokémon Go, see who players are out and about with, and examine associations with social connectedness. Mental health researchers could investigate the effects of Pokémon Go on symptoms of depression.

Additionally, collaborating with game designers would help researchers navigate the relatively poor funding landscape in public health. Pokémon Go was developed by Niantic, a software development company that was originally a start-up within Google, together with Nintendo and the Pokémon Company. Since the costs associated with developing and testing new games are immense, it is important to recognise the possible co-benefits for game makers and researchers in working together and making the most of their respective capacities.

\section{Public health is too slow}

An overarching issue that stymies public health research in this sector is the relatively slow speed at which researchers operate to rigorously design studies, collect and interpret data, and prepare a peer-reviewed paper. By the time a randomised controlled trial with objective measures is set up to investigate the health effects of Pokémon Go, the game's popularity may well have passed, and the game may have been superseded by the next craze. The rapid rise and fall of a game's popularity is salient to game makers; does anyone remember Angry Birds?

Public health stakeholders also need to reconsider how health-related gaming is marketed and promoted. This Twitter comment nicely sums up how public health would have ruined the Pokéman Go game with poor branding: "Man, @Nintendo made the right call with \#PokémonGo instead of the original title: Get Some Fresh Air and Exercise". ${ }^{2}$ 


\section{Corporations that harm health exploit popular trends}

Even if Pokémon Go, with all the associated media hype, is a quick-lived fad, it may signal the beginning of a new genre of apps and games that lead to unanticipated health outcomes. If public health stakeholders want to capitalise on this genre, it is essential that more meaningful partnerships across health, information technology, marketing and communications are formed. Some companies that endanger public health quickly used the Pokémon Go trend as an opportunity to promote consumption of fast food. ${ }^{13}$ Public health agencies need to be just as nimble and responsive, or forever be creating games that nobody plays.

\section{Acknowledgements}

JC holds a postdoctoral fellowship that is funded by the Heart Foundation.

\section{Competing interests}

BF has received money from the World Health Organization; the Sax Institute; Cancer Council NSW; NSW Health; the Union for International Cancer Control; and the Asian Center for WTO \& International Health Law and Policy, National Taiwan University, College of Law.

\section{Author contributions}

All three authors conceived of the paper and contributed to the writing.

\section{References}

1. Abad-Santos A. Pokémon Go: 9 questions about the game you were too embarrassed to ask. Vox. 2016 Jul 16 [cited 2016 Jul 25]. Available from: www.vox. com/2016/7/12/12158372/pokemon-go-ios-android-gamequestions

2. Grubb J. Pokémon Go is at 75 million downloads as it sets more records. Venture Beat. 2016 Jul 25 [cited 2016 Jul 25]. Available from: venturebeat.com/2016/07/25/ pokemon-go-is-at-75-million-downloads-as-itsets-more-records
3. Perez S. Pokémon Go tops Twitter's daily users, sees more engagement than Facebook. Tech Crunch. 2016 Jul 13 [cited 2016 Jul 25]. Available from: techcrunch. com/2016/07/13/pokemon-go-tops-twitters-daily-userssees-more-engagement-than-facebook

4. Angry Aussie, @angryaussie. I started using \#pokemongo seriously on Friday. Can you tell? 2016 Jul 11 [cited 2016 Jul 25]. Available from: www.instagram.com/p/ BHsvS57gWEJ

5. Gertler I, @iangertler. Evening stroll in the park with the family-capturing \#Pokemon creatures with dozens of others! Twitter. 2016 Jul 24 [cited 2016 Nov 2]. Available from: twitter.com/lanGertler/status/757378713415741440

6. Gotta walk 'em all: an animal shelter is asking Pokémon Go players to walk its dogs. Stylist: Life. 2016 Jul 15 [Cited 2016 Jul 25]. Available from: pages.stylist.co.uk/ life/pokemon-go-game-muncie-animal-shelter-indianarescue-dogs-walk-volunteer-technology-gaming

7. Grayson N. Pokemon Go helped me cope with my social anxiety. Kotaku. 2016 Jul 20 [cited 2016 Jul 25]. Available from: kotaku.com/pokemon-go-helped-me-cope-with-mysocial-anxiety- 1783988220

8. Pfaeffli Dale L, Dobson R, Whittaker R, Maddison R. The effectiveness of mobile-health behaviour change interventions for cardiovascular disease self-management: a systematic review. Eur J Prev Cardiol. 2016;23(8):801-17.

9. Wang JB, Cadmus-Bertram LA, Natarajan L, White MM, Madanat H, Nichols JF, et al. Wearable sensor/device (Fitbit One) and SMS text-messaging prompts to increase physical activity in overweight and obese adults: a randomized controlled trial. Telemed J E Health. 2015;21(10):782-92.

10. Althoff T, White RW, Horvitz E. Influence of Pokémon Go on physical activity: study and implications. J Med Internet Res. 2016;18(2):e315.

11. Riley WT, Rivera DE, Atienza AA, Nilsen W, Allison SM, Mermelstein R. Health behavior models in the age of mobile interventions: are our theories up to the task? Transl Behav Med. 2011;1(1):53-71.

12. Held J, @josephcurrency. Man, @Nintendo made the right call with \#PokemonGo instead of the original title: Get Some Fresh Air and Exercise. Twitter. 2016 Jul 11 [cited 2016 Jul 25]. Available from: twitter.com/josephcurrency/ status/752677723995197440

13. Russell J. Pokémon Go finally goes live in Japan with McDonald's the first sponsored location. TechCrunch. 2016 Jul 21 [cited 2017 Mar 2]. Available from: techcrunch. com/2016/07/21/pokemon-go-is-finally-available-in-japan

\section{Copyright: (c) (7)(2) (2)}

(C) 2017 Freeman et al. This article is licensed under the Creative Commons Attribution-NonCommercial-ShareAlike 4.0 International Licence, which allows others to redistribute, adapt and share this work non-commercially provided they attribute the work and any adapted version of it is distributed under the same Creative Commons licence terms. See: www.creativecommons.org/licenses/by-nc-sa/4.0/ 